\title{
A Framework for Anomaly Detection in Activities of Daily Living using an Assistive Robot
}

\author{
Salisu Wada Yahaya, Ahmad Lotfi, Senior Member, IEEE, Mufti Mahmud, Senior Member, IEEE
}

\begin{abstract}
This paper presents an overview of an ongoing research to incorporate an assistive robotic platform towards improved detection of anomalies in daily living activities of older adults. This involves learning human daily behavioural routine and detecting deviation from the known routine which can constitute an abnormality. Current approaches suffer from high rate of false alarms, therefore, lead to dissatisfaction by clients and carers. This may be connected to behavioural changes of human activities due to seasonal or other physical factors. To address this, a framework for anomaly detection is proposed which incorporates an assistive robotic platform as an intermediary. Instances classified as anomalous will first be confirmed from the monitored individual through the intermediary. The proposed framework has the potential of mitigating the false alarm rate generated by current approaches.
\end{abstract}

\section{INTRODUCTION}

The world population of older adults (i.e. people between the age of 65 and above) is increasing and it is estimated to be over 1.92 billion in 2050 [1]. This leads to increase in cost of care for the senior citizens. Moreover, study has shown that these senior citizens prefer to live in their own homes rather than being looked after in a care homes [2] [3]. Given the health-related challenges associated with older people, systems are set in place to monitor the Activities of Daily Living (ADL) in order to promote the independent living and improve the quality of life for older adults. ADL are those activities an individual must be able to perform in order to be able to live independently such as eating, maintaining personal hygiene and continence, mobility etc. Abnormalities in these activities can be an indication of health decline or challenges related to Mild Cognitive Impairment (MCI) such as Dementia, which are detrimental to wellbeing.

The current approaches to anomaly detection in ADL suffer from high rate of false alarms making the system unreliable, and therefore, leads to dissatisfaction by carers and clients [4]. This may be connected to the dynamic nature of human activity which is subject to changes based on seasonal or other physical factors. To build a reliable anomaly detection system and reduce the rate of false alarm, the user's behaviour must be modelled accurately by taking into account the changes in the individual behavioural routine.

This can be achieved by building a computational model that can adapt to changes in human behaviour. Using a robotic platform as an intermediary in the anomaly detection system is proposed in this paper. Activities classified as anomalous will be queried through the robotic platform for confirmation from

S. W. Yahaya is PhD student at the Dept. of Computing \& Technology, School of Science \& Technology, Nottingham Trent University, Clifton, Nottingham, NG11 8NS, UK (e-mail: salisu.yahaya2015@my.ntu.ac.uk).

A. Lotfi is a Professor of Computational Intelligence at the School of Science \& Technology, Nottingham Trent University, Clifton, Nottingham, the user. This will enable the model to obtain the user's feedback in real time before making its final prediction.

\section{ANOMALY DETECTION IN ACTIVITIES OF DAILY LIVING}

In the context of ADL, anomaly is any significant deviation from individual's usual behavioural routine. Research has been conducted to detect abnormality in ADL using different computational methodologies. Hoque et. al. [5] developed an ADL anomaly detecting system termed "Holmes" using DBSCAN clustering. Similarly, the duration of performed activities, time and number of sensor events are extracted and clustered using DBSCAN in [6]. Instance with unusual duration or irregular sensor events are classified as anomalous. A method based on detecting temporal relation between activities is used for detecting anomaly in [7]. Since human behavioural routine varies from one individual to another, the most common approach is to model the behaviour of an individual to serve as a baseline. Subsequent behavioural routines are compared to the baseline and deviations are classified as anomalies. The same approach is applied in [8] with One-Class Support Vector Machine (OC-SVM) to detect abnormality in sleeping pattern. While these approaches are able to detect anomalous instance of activities, they are not flexible to accommodate changes in user's behavioural routine that are not anomalous. This may be due to the lack of a feedback mechanism.

\section{ROBOTS FOR SOCIAL INTERACTIONS}

Robots for social interactions also known as Socially Assistive Robots (SAR) are robots that provides assistance via social means (i.e. through interactions) rather than through physical means [9]. In recent years, researchers have been applying SAR for various purposes ranging from health care monitoring, social engagement and as motivational agent. For example, in [10] an exercise trainer robot is built to coach and motivate older adults to perform physical activities. The robot uses Kinect sensor to analyse human pose and gives feedback in form of facial expression and speech. Similarly, a companion robot for people living with MCI is proposed in [11]. This robot assists with keeping track of reminders (e.g. taking medication), administering cognitive exercises and establishing communication with relatives and carers. A research is conducted in [12] using an SAR to help children

NG11 8NS, UK (phone: +44 (0)115 848 8390; e-mail: ahmad.lotfi@ntu.ac.uk).

M. Mahmud is a (Senior) Lecturer of Computing \& Technology, School of Science \& Technology, Nottingham Trent University, Clifton, Nottingham, NG11 8NS, UK (e-mail: mufti.mahmud@ \{ntu.ac.uk,gmail.com\}). 


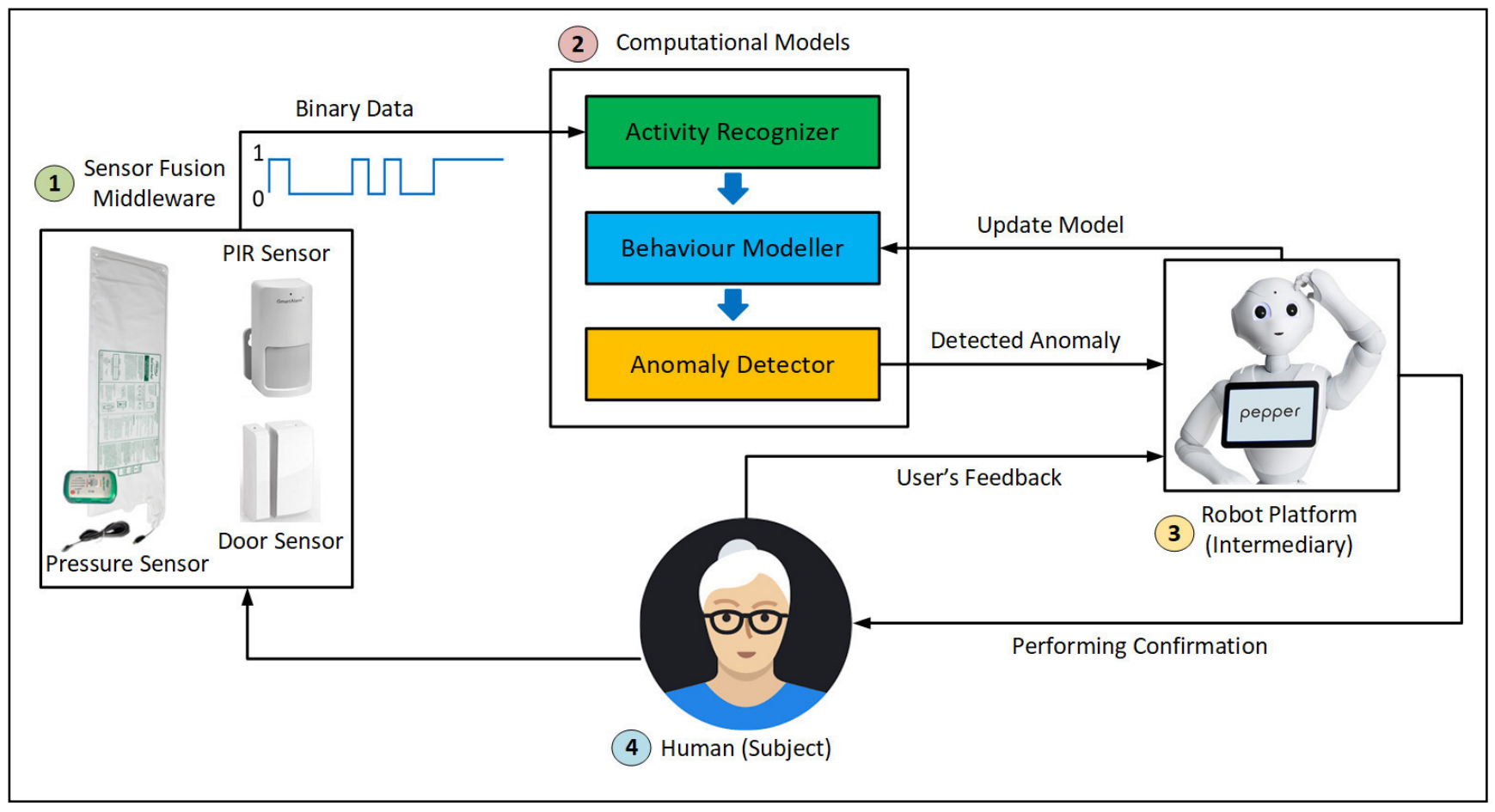

Figure 1. Proposed architecture for anomaly detection in ADL.

with diabetes learn how to manage the condition while in [13], a robot is used to teach children living with Autism Spectrum Condition (ASC) how to see things from a different person's perspective which is also known as Visual Perspective Taking (VPT).

Taking advantage of the potential of these devices in home settings, as well as robot's support for multi-modal interaction through gesture, touch and speech, they can serve as a good intermediary for an anomaly detection system. Moreover, studies have shown that the physical embodiment of robots facilitates Human-Robot Interaction (HRI) [14] [15].

\section{Proposed ARCHITECTURE FOR ANOMALY DETECTION}

A schematic diagram is shown in Figure 1 of the proposed framework for anomaly detection consisting of four interconnected layers, namely; Sensor Fusion, Computational Models, Intermediary and Human layer. The Computational Models layer has three components; Activity Recogniser, Behaviour Modeller and Anomaly Detector.

The Sensor Fusion layer consists of a network of interconnected low-level sensors for data collection. These sensors include Passive Infrared (PIR) Motion Sensors in the various location of the house, Pressure sensor on bed and on chair, Door sensor on the door etc. This is the most widely acceptable sensing modality due to its non-invasive nature compared to vision based approached which raises privacy concerns [2]. The data generated by these sensors are binary in nature with 0 and 1 signifying inactive and active states respectively. The data is then sent to the Computational Model layer for processing.

The Activity Recognizer component interprets the binary data into readable human activities. Rule-based approaches are used for the recognition. The recognized activities involve eating, preparing meal, toileting, sleeping etc. Each activity is accompanied by its start time, end time, and location as shown in Table 1.

TABLE I. SAMPLE DATA FOR ACTIVITIES OF DAILY LIVING.

\begin{tabular}{|l|l|l|l|}
\hline \multicolumn{1}{|c|}{ Start Time } & \multicolumn{1}{|c|}{ End Time } & \multicolumn{1}{c|}{ Activity } & \multicolumn{1}{c|}{ Location } \\
\hline $\begin{array}{l}2018-05-01 \\
17: 19: 31\end{array}$ & $\begin{array}{l}2018-05-01 \\
17: 28: 45\end{array}$ & Eating & Dining Room \\
\hline $\begin{array}{l}2018-05-01 \\
17: 28: 59\end{array}$ & $\begin{array}{l}2018-05-01 \\
20: 34: 31\end{array}$ & Toileting & Toilet \\
\hline $\begin{array}{l}2018-05-01 \\
22: 49: 43\end{array}$ & $\begin{array}{l}2018-05-02 \\
07: 46: 07\end{array}$ & Sleeping & Bedroom \\
\hline
\end{tabular}

The Behavioural Modeller component builds a model of the person's behavioural routine. Apart from our recent work in [8], other computational models such as those capable of learning temporal and sequence data such as Hidden Markov Model (HMM) and Recurrent Neural Network (RNN) will be explored.

The Anomaly Detector component compares the subsequent user's behaviour against the model build by the Behaviour Modeller. Deviation from the built model that can be an indication of abnormality will be sent to the Intermediary layer (robot) for confirmation.

The Intermediary layer serves as a bridge between the Computational Models layer and the Human. The robot platform will have a bi-direction communication capability to enable it to interact with humans. It will confirm activities that are classified as abnormal by the Anomaly Detector component. The user's response will be fed back to the Behaviour Modeller. In a situation where the user's response goes contrary to the prediction of the Anomaly Detector over a 


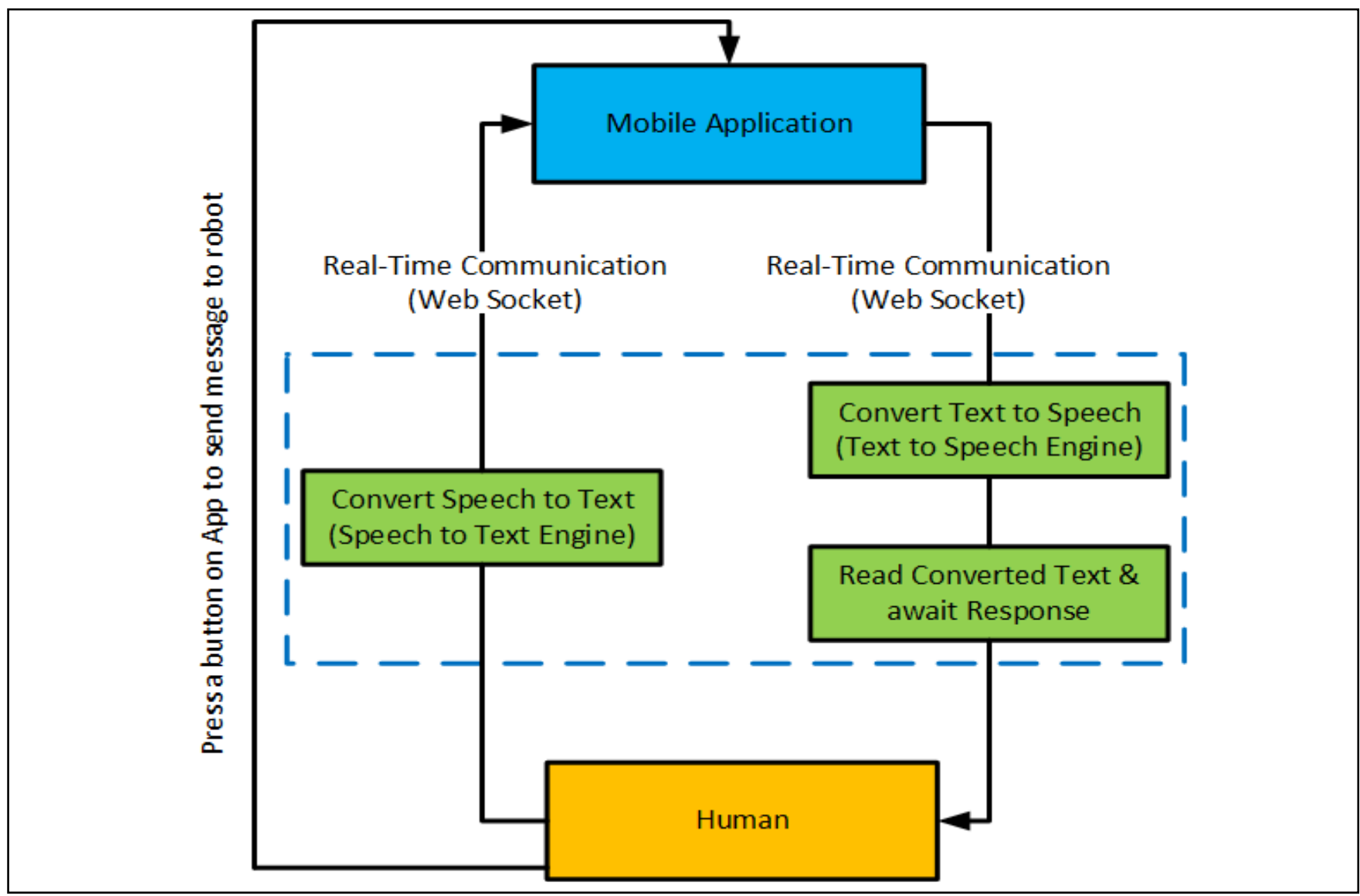

Figure 2. Implementation diagram for real-time human-robot communication.

certain threshold, the user's response along with the model's prediction will be forwarded for an expert evaluation. The expert, in this case, can be the carer or medical personnel. The expert's opinion is given more weight and serves as the final prediction. Important design guidelines for HRI such as error prevention and correction, consistency, user control and freedom [16] [17] will be taken into consideration as well as the concept of persuasive psychology in order to increase the acceptability of the platform and make it less invasive. To make the proposed framework more robust, computational models based on the concept of active learning will also be explored. This will enable the Behaviour Modeller to learn in a progressive manner based on the feedback received from the Human.

\section{EVALUATION Strategy}

The different layers of the proposed anomaly detection framework will be evaluated. The ability of the Sensor Fusion middleware to aggregate the heterogenous sensors for data collection will be evaluated. At the Computational Model layer, the evaluation will focus primarily on the system's ability to model the individual's behavioural routine and detects abnormalities in it. Publicly available ADL datasets as well as experimental data collected specifically for this research will be utilized. Different anomalous instance will be simulated to test the robustness of the computational models.

Evaluation of the Intermediary layer will focus on HRI to assess the acceptability of the platform for behaviour monitoring. Devices capable of serving as an intermediary such as mobile phones and screen-only based interface will be tested. While assistive robots provide the advantage of physical embodiment and support for multi-modal interaction, factors such as the cost of the platform, trust-related issues and technical difficulties might make other intermediaries more suitable. The monitored individuals, carers and experts will be incorporated in the evaluation of the proposed architecture.

To test for the feasibility of using a robot platform as an intermediary, an application is implemented with a bidirectional communication capability using Pepper robot. Pepper is a humanoid robot from SoftBank Robotics equipped with a touch screen display and wheels for mobility. It is capable of interacting with humans, recognizing faces and basic human emotions [18].

The implementation diagram for real-time human-robot communication is illustrated in Figure 2. A mobile application is built to simulate the Anomaly Detector component. A user presses a button on the app to send a message to the robot similar to how the Anomaly Detector will broadcast an anomalous activity to the intermediary. The generated message is transmitted via web socket in real-time to the robotic platform. The Robot uses a Text to Speech engine to convert the message to an audio format, reads it out and waits for the user's feedback. The user's response in form of speech 
is converted to text using Speech to Text engine. The converted text is then retransmitted via web socket to the mobile application in real-time. This is a simulation of the intermediary component without the Computational Models and Sensor Fusion layers.

\section{CONCLUSION}

In this paper, a framework for anomaly detection in ADL incorporating a SAR platform is presented. The platform serves as an intermediary between the anomaly detection model and the human for performing confirmation of detected anomalies in order to reduce the rate of false alarm affecting the current approaches. Using computational models based on active learning, an anomaly detection system can be built that can adapt to changes in individual's behaviour routine with less false alarm. Future work will include testing and deployment of the complete system in a real home environment.

\section{REFERENCES}

[1] S. Chernbumroong, S. Cang, A. Atkins and H. Yu, "Elderly Activities Recognition and Classification for Applications in Assisted Living," Expert Systems with Applications, vol. 40, pp. 1662 - 1674, 2013.

[2] P. Rashidi and A. Mihailidis, "A Survey on AmbientAssisted Living Tools for Older Adults," IEEE Journal of Biomedical and Health Informatics, vol. 17, pp. 579-590, 2013.

[3] A. Lotfi, C. Langensiepen, S. Mahmoud and M. J. Akhlaghinia, "Smart homes for the elderly dementia sufferers: identification and prediction of abnormal behaviour," Journal of Ambient Intelligence and Humanized Computing, vol. 3, pp. 205-218, 2012.

[4] K. Z. Haigh, L. M. Kiff and G. Ho, "The Independent LifeStyle Assistant: Lessons Learned," Assistive Technology, vol. 18, pp. 87-106, 2006.

[5] E. Hoque, R. F. Dickerson, S. M. Preum, M. Hanson, A. Barth and J. A. Stankovic, "Holmes: A Comprehensive Anomaly Detection System for Daily In-home Activities," in 2015 International Conference on Distributed Computing in Sensor Systems, 2018.

[6] L. G. Fahad and R. M., "Anomalies Detection in Smart-Home Activities," in 2015 IEEE 14th International Conference on Machine Learning and Applications (ICMLA), 2015.

[7] V. Jakkula, D. J. Cook and A. S. Crandall, “Temporal pattern discovery for anomaly detection in a smart home," in 2007 3rd IET International Conference on Intelligent Environments, 2007.

[8] S. W. Yahaya, C. Langensiepen and A. Lotfi, "Anomaly Detection in Activities of Daily Living Using One-Class Support Vector Machine," in 18th UK Workshop on Computational Intelligence, 2018.
[9] D. Feil-Seifer and M. J. Mataric, "Defining socially assistive robotics," in 9th International Conference on Rehabilitation Robotics, 2005.

[10] A. Lotfi, C. Langensiepen and S. W. Yahaya, "Socially Assistive Robotics: Robot Exercise Trainer for Older Adults," Technologies, vol. 6, 2018.

[11] H. -M. Gross, C. Schroeter, S. Mueller, M. Volkhardt, E. Einhorn, A. Bley, T. Langner, C. Martin and M. Merten, "I'll keep an eye on you: Home robot companion for elderly people with cognitive impairment," in 2011 IEEE International Conference on Systems, Man, and Cybernetics, 2011.

[12] L. Cañamero and M. Lewis, "Making New "New AI" Friends: Designing a Social Robot for Diabetic Children from an Embodied AI Perspective," International Journal of Social Robotics, vol. 8, pp. 523--537, 2016

[13] L. Wood, B. Robins, G. Lakatos, D. S. Syrdal, Z. Abolfazl and D. Kerstin, "Utilising humanoid robots to assist children with autism learn about Visual Perspective Taking," in UK Robotics and Autonomous Systems Conference, 2017.

[14] J.-J. Cabibihan, H. Javed, M. J. Ang and S. M. Aljunied, "Why Robots? A Survey on the Roles and Benefits of Social Robots in the Therapy of Children with Autism," International Journal of Social Robotics, vol. 5, pp. 593-618, 2013.

[15] J. Wainer, D. J. Feil-seifer, D. A. Shell and M. J. Mataric, "The role of physical embodiment in humanrobot interaction," in 15th IEEE International Symposium on Robot and Human Interactive Communication, Hatfield, 2006.

[16] C. Breazeal, "Social interactions in HRI: the robot view," IEEE Transactions on Systems, Man, and Cybernetics, Part C (Applications and Reviews), vol. 34, no. 2, pp. 181-186, 2004.

[17] O. M. -.. E. Sucar, S. H. Aviles and C. Miranda-Palma, "From HCI to HRI - usability inspection in multimodal human - robot interactions," in 12th IEEE International Workshop on Robot and Human Interactive Communication, 2003.

[18] SoftBank, "Pepper the humaoid robot," SoftBank Robotics, [Online]. Available: https://www.softbankrobotics.com/emea/en/pepper. [Accessed 0301 2019]. 